\title{
Orientation-Specific Visual Evoked Potential Deficits in Multiple Sclerosis
}

\author{
STUART G. COUPLAND and TREVOR H. KIRKHAM
}

SUMMARY: Checkerboard pattern reversal visual evoked potentials (VEPS) have proved useful in the confirmation of optic nerve disease in patients with multiple sclerosis $(M S)$. Recently, evidence of orientation-specific loss in contrast sensitivity and the presence of orientationspecific visual evoked potential (VEP) deficits in MS patients has been obtained using sinusoidal gratings as stimuli. This study reports the presence of orientationspecific VEP delay in MS using the conventional checkerboard pattern presented in two orientations: normally oriented (check condition) or diagonally oriented (diamond condition).

RESUMÉ: La valeur de l'enregistrement des potentiels évoqués visuels (PEVs) aux renversements de patrons dans la sclérose en plaques (SEP) est bien établie dans la littérature. Utilisant comme stimulation les barres sinusoidales, des études récentes ont démontré des anomalies des PEVS et de la sensibilité de contraste dépendant de l'orientation du stimulus. Nous décrivons ici les délais des PEVS aux renversements de patrons qui dépendaient de l'orientation du stimulus, soit aux carreaux, soit aux
Peak latency values of the N70 and PIOO components of the VEP were statistically analyzed using appropriate ANOVA and nonparametric statistics. As a group $M S$ patients showed significant VEP delays under check and diamond pattern conditions. However, individual subject analysis revealed that about $20 \%$ of the MS population show VEP delay to only one pattern orientation. It was shown that by including a diamond pattern condition the diagnostic yield of VEP delay in these clinically definite MS patients was increased $11 \%$ over that obtained with check stimulation alone.

diamants. Les analyses statistiques de pics N7O et P100 ont été accomplies utilisant l'ANOVA et les tests non-paramétriques. Dans $20 \%$ des malades avec la SEP des délais des PEVs spécifiques pour la stimulation soit aux carreaux, soit aux diamants, ont été découverts. En ajoutant la stimulation aux diamants à notre examen nous avons augmenté de $10 \%$ le nombre d'anomalies détectables des PEVS dans notre population de malades souffrant de la $S E P$.

\footnotetext{
From the Departments of Neurology, Neurosurgery and Ophthalmology, McGill University, and the Department of Neuro-Ophthalmology at the Montreal Neurological Institute.

Requests for reprints to: Dr. T.H. Kirkham, Department of Neuro-Ophthalmology, Montreal. Neurological Hospital, 3801 University \#201; Montreal, Quebec, Canada. H3A 2B4.
}

\begin{abstract}
INTRODUCTION
Over the past decade the visual evoked potential (VEP) has become a routine clinical measure for assessing optic nerve and visual pathway function in patients with clinically definite or suspected multiple sclerosis (MS). It has been established that the VEPs elicited by checkerboard patternreversal are superior to the flash VEPs in the detection of optic nerve lesions. Systematic investigation of checkerboard pattern parameters such as check size, contrast and reversal rate have been conducted to determine the optimal stimulus conditions for detecting optic nerve dysfunction. Some researchers (Bodis-Wollner et al., 1979; Ochs et al., 1980) have used different patterns including bar gratings or concentric circular gratings to elicit pattern VEPs to determine whether some pattern stimuli are more clinically useful than others.
\end{abstract}

Regan et al. (1980) recently reported a small group of MS patients in whom orientation-specific losses of contrast sensitivity at different spatial frequencies were detected. Independent evidence of orientation-specific VEP deficits in MS patients was obtained by Camisa et al (1981) using gratings as stimuli. They reported finding VEP delays which were dependent upon the orientation of the grating stimulus. These findings suggested that for the diagnosis of visual system involvement in MS, patterns or gratings of different orientation or of different spatial frequency should be used in order to increase the diagnostic yield of the test procedure. We therefore studied a group of multiple sclerosis patients to look for evidence of orientation-specific deficits in pattern reversal visual evoked potentials. We used conventional square checkerboard stimuli and then, with the same electrode recording placement, recorded the VEP to a diamond shaped pattern which was ob- 
tained by rotating the CRT stimulator 45 degrees thereby changing the orientation of pattern edge while leaving the spatial averaged luminance of the pattern unchanged.

\section{MATERIALS AND METHODS \\ Patients}

We examined 44 normal controls and 105 patients who had clinically definite MS as defined by the McDonald and Halliday (1977) criteria. These same patients were used in a study of flash electroretinogram (ERG) abnormalities in multiple sclerosis (Coupland and Kirkham, 1982). As in the previous study the patients were divided into 4 groups as follows: 1) 31 patients in whom there was no clinical evidence for optic nerve disease (designated as MS "Normals"), 2) 8 patients with clinical evidence of unilateral optic nerve disease (OND) of the left eye, 3) 12 patients with OND of the right eye, and 4) 54 patients with a history or clinical evidence of bilateral OND. The clinical criteria for optic nerve disease were the same as those used in our previous studies (Kirkham and Coupland 1981; Coupland and Kirkham, 1982).

\section{Stimulation and Recording Methods}

VEPs were recorded to a 11 degree circular test field containing patternreversing checkerboard or diamond patterns (pattern size $=30$ '). The luminances of the bright and dark checks were $200 \mathrm{~cd} / \mathrm{m}^{2}$ and $10 \mathrm{~cd} / \mathrm{m}^{2}$ respectively (approximately $80 \%$ contrast) as determined by a Hagner S-2 Universal Spot Photometer (Optikon Corp., Waterloo, Ont.). Diamond stimulation was obtained by rotating the CRT stimulator 45 degrees so that the spatial averaged luminance of the stimulating pattern was unchanged. VEPs were recorded over $0_{1}$ and $\mathrm{O}_{2}$ with active sites referenced to linked mastoids. The raw EEG was amplified $50,000 \mathrm{x}$ by Grass $\mathrm{P} 5-11 \mathrm{~J}$ preamplifiers (Grass Instruments, Quincy, Mass.) with a .1 - $300 \mathrm{~Hz}$. bandwidth and 128 samples were signal averaged by an LSI-11 microprocessor-based TN1710 multichannel analyzer (TracorNorthern Instruments, Wisconsin) and the resulting averaged VEP waveforms were stored on floppy disk for off-line analysis.

VEP records were scored by visual inspection and the peak latency of the early negative (N70) and major positive (P100) components were determined for each monocular checkerboard and diamond condition. Using our normative sample mean and standard deviation a criterion of latency delay exceeding $99 \%$ of normal controls was determined. In addition, the absolute N70-P100 interpeak latency difference was used as an index of temporal dispersion of the VEP waveform. Peak latency values of checkerboard and diamond conditions for normal and multiple sclerosis populations were statistically analyzed using appropriate
ANOVA and nonparametric statistics. Within the multiple sclerosis population the relative frequency of "normal" and "abnormal" component latencies under check or diamond pattern stimulation were tabulated and tested using the McNemar chi-square.

\section{RESULTS}

Mean peak latencies of $\mathrm{N} 70$ and P100 as well as mean N70-P100 interpeak latency differences measured under all recording conditions are presented for the normal control subjects and the four MS groups in Tables 1 and 2. It is clear that for all four MS groups the mean and standard deviation of peak latency of N70 and P100 was greater than that of the normal

TABLE 1

Mean latencies for N7O and PIOO components on Check and Diamond conditions

\begin{tabular}{|c|c|c|c|c|}
\hline \multicolumn{5}{|c|}{ CHECK Stimulation } \\
\hline & \multicolumn{2}{|c|}{ LEFT EYE } & \multicolumn{2}{|c|}{ RIGHT EYE } \\
\hline Group & $\begin{array}{c}0_{1} \text { N70 } \\
\text { Mean } \pm \text { sd }\end{array}$ & $\begin{array}{c}\text { 0.P100 } \\
\text { Mean } \pm \text { sd }\end{array}$ & $\begin{array}{c}0_{1} \text { N70 } \\
\text { Mean } \pm \text { sd }\end{array}$ & $\begin{array}{c}0, P 100 \\
\text { Mean } \pm \text { sd }\end{array}$ \\
\hline MS normal & $78.1 \pm 14.4$ & $109.8 \pm 15.3$ & $78.1 \pm 12.4$ & $111.2 \pm 13.7$ \\
\hline Left OND & $92.3 \pm 23.8+$ & $122.8 \pm 26.1+$ & $86.6 \pm 21.2$ & $112.6 \pm 21.2$ \\
\hline Right OND & $80.1 \pm 17.9$ & $112.3 \pm 14.2$ & $85.3 \pm 16.5^{*}$ & $122.4 \pm 14.3+$ \\
\hline Bilat OND & $93.7 \pm 21.9+$ & $128.5 \pm 24.5 \$$ & $92.4 \pm 23.6+$ & $127.9 \pm 25.8 \$$ \\
\hline NORMALS & $68.8 \pm 5.9$ & $98.5 \pm 4.5$ & $67.8 \pm 5.6$ & $97.4 \pm 5.2$ \\
\hline Group & $\begin{array}{c}\mathbf{0}_{2} \mathbf{N} 70 \\
\text { Mean } \pm \text { sd }\end{array}$ & $\begin{array}{c}02 \text { P } 100 \\
\text { Mean } \pm \text { sd }\end{array}$ & $\begin{array}{c}0_{2} N 70 \\
\text { Mean } \pm \text { sd }\end{array}$ & $\begin{array}{c}02 P 100 \\
\text { Mean } \pm \text { sd }\end{array}$ \\
\hline MS normal & $78.7 \pm 14.3$ & $110.3 \pm 15.2^{*}$ & $78.9 \pm 13.0$ & $111.7 \pm 16.8^{*}$ \\
\hline Left OND & $91.4 \pm 19.9+$ & $121.8 \pm 25.5+$ & $82.1 \pm 21.2$ & $110.5 \pm 20.3$ \\
\hline Right OND & $78.4 \pm 17.0$ & $111.0 \pm 16.8$ & $84.1 \pm 19.8^{*}$ & $123.1 \pm 16.3+$ \\
\hline Bilat OND & $93.8 \pm 20.5^{*}$ & $129.8 \pm 22.8 \$$ & $93.4 \pm 25.2+$ & $129.7 \pm 26.0 \$$ \\
\hline NORMALS & $68.3 \pm 6.0$ & $98.2 \pm 5.1$ & $68.0 \pm 5.3$ & $97.5 \pm 5.7$ \\
\hline \multicolumn{5}{|c|}{ DIAMOND Stimulation } \\
\hline Group & $\begin{array}{c}01 N 70 \\
\text { Mean } \pm \text { sd }\end{array}$ & $\begin{array}{c}\text { 01 P100 } \\
\text { Mean } \pm \text { sd }\end{array}$ & $\begin{array}{c}\text { 0. N70 } \\
\text { Mean } \pm \text { sd }\end{array}$ & $\begin{array}{c}01 P 100 \\
\text { Mean } \pm \text { sd }\end{array}$ \\
\hline MS normal & $76.0 \pm 12.9$ & $110.2 \pm 14.0$ & $78.1 \pm 16.5$ & $112.5 \pm 15.7^{*}$ \\
\hline Left OND & $84.1 \pm 14.9^{*}$ & $123.3 \pm 19.6+$ & $77.0 \pm 17.8$ & $104.2 \pm 18.3$ \\
\hline Right OND & $79.5 \pm 16.3$ & $113.3 \pm 16.1$ & $87.4 \pm 14.7+$ & $124.5 \pm 17.0+$ \\
\hline Bilat OND & $86.7 \pm 20.7+$ & $124.9 \pm 24.8 \$$ & $90.9 \pm 19.8+$ & $128.5 \pm 21.1 \mathrm{~S}$ \\
\hline NORMALS & $67.2 \pm 6.4$ & $99.3 \pm 4.3$ & $67.3 \pm 5.3$ & $99.4 \pm 4.7$ \\
\hline Group & $\begin{array}{c}\mathbf{0}_{2} \text { N70 } \\
\text { Mean } \pm \text { sd }\end{array}$ & $\begin{array}{c}02 \text { P } 100 \\
\text { Mean } \pm \text { sd }\end{array}$ & $\begin{array}{c}0_{2} \text { N70 } \\
\text { Mean } \pm \text { sd }\end{array}$ & $\begin{array}{c}02 \text { P } 100 \\
\text { Mean } \pm \text { sd }\end{array}$ \\
\hline MS normal & $78.5 \pm 16.5$ & $111.2 \pm 15.1^{*}$ & $78.6 \pm 17.7$ & $114.5 \pm 18.7+$ \\
\hline Left OND & $80.5 \pm 18.7$ & $118.0 \pm 19.7^{*}$ & $83.9 \pm 14.8$ & $106.6 \pm 17.2$ \\
\hline Right OND & $81.7 \pm 17.4$ & $111.4 \pm 13.9$ & $85.2 \pm 17.6+$ & $121.0 \pm 12.6+$ \\
\hline Bilat OND & $88.7 \pm 20.0+$ & $126.9 \pm 24.1 \$$ & $92.2 \pm 20.4+$ & $128.9 \pm 21.1 \$$ \\
\hline NORMALS & $67.1 \pm 6.5$ & $98.1 \pm 5.7$ & $67.3 \pm 5.6$ & $99.0 \pm 5.4$ \\
\hline
\end{tabular}

Scheffé procedure: $\quad *=p<.05 \quad+=p<.01 \quad \$=p<.001$ 
TABLE 2

Absolute Mean N70-P100 latency differences for Check and Diamond conditions

\section{CHECK Stimulation}

LEFT EYE

01 N70-P 100

$\begin{array}{ll}\text { Group } & \text { Mean } \pm \text { sd } \\ \text { MS normal } & 31.6 \pm 11.5 \\ \text { Left OND } & 30.5 \pm 9.2 \\ \text { Right OND } & 32.3 \pm 7.4 \\ \text { Bilat OND } & 34.8 \pm 15.4 \\ \text { NORMALS } & 29.6 \pm 5.2\end{array}$

O2 N70-P 100

Group

MS normal

Left OND

Right OND

Bilat OND

NORMALS

\section{RIGHT EYE}

01 N70-P 100

Group
MS normal
Left OND
Right OND
Bilat OND
NORMALS

- Group

MS normal

Left OND

Right OND

Bilat OND

NORMALS

02 N70-P 100

\section{Mean $\pm s d$ \\ $33.1 \pm 12.9$ \\ $36.1 \pm 11.1$ \\ $37.2 \pm 11.6$ \\ $35.5 \pm 10.8^{*}$ \\ $29.6 \pm 5.4$}

Mean \pm sd
$32.8 \pm 14.5$
$32.9 \pm 9.6$
$39.1 \pm 13.2$
$36.3 \pm 12.6^{*}$
$29.6 \pm 5.8$

01 N70-P100

01 N70-P 100

Group
MS normal
Left OND
Right OND
Bilat OND
NORMALS

02 N70-P 100

\section{Group}

MS normal

Left OND

Right OND

Bilat OND

NORMALS

$$
\begin{aligned}
& \text { Mean } \pm s d \\
& 34.5 \pm 9.9 \\
& 36.8 \pm 4.3 \\
& 35.1 \pm 5.6 \\
& 38.3 \pm 11.3^{*} \\
& 32.0 \pm 6.7
\end{aligned}
$$

Group
MS normal
Left OND
Right OND
Bilat OND
NORMALS

Group

MS normal

Left OND

Right OND

Bilat OND

NORMALS

\section{DIAMOND Stimulation}

that the MS left OND group mean differs significantly from the normal control group only under left monocular stimulation conditions and does not differ significantly from the normal for right monocular stimulation. Similarly the MS right OND group demonstrates significantly greater mean $\mathrm{N} 70$ peak latency only for right monocular stimulation conditions. The MS bilateral OND group mean N70 component peak latency is significantly longer than normal to stimulation of either eye. The mean N70 peak latency of the "MS normals" group is not significantly greater than normal for either eye. For all four MS groups the same pattern of occurrence of VEP delay is seen for both check and diamond pattern orientations.

\section{Comparisons between $M S$ groups and normals: $P 100$}

The P 100 peak latencies from the four MS groups were compared with the normal control group using the Scheffé procedure (Table 1). The mean P 100 peak latency of the MS left OND group was significantly delayed for left monocular stimulation but was not significantly different from the normal control group mean for right monocular stimulation. This finding was consistent across check and diamond pattern orientation. Likewise the mean P100 peak latency for the MS right OND group was significantly delayed only for stimulation of the affected eye. The MS bilateral OND group show significantly delayed mean P100 peak latency to monocular stimulation of either eye. Interestingly the mean P100 peak latency for the "MS normals" was also significantly delayed in 6 of the 8 recording conditions (Table 1). Again for all four MS groups the same pattern of occurrence of VEP delay was seen regardless of pattern orientation.

\section{Paired Comparisons between MS groups and normals: N70-P100}

The N70-P100 mean absolute interpeak latency difference for the MS bilateral OND group was significantly greater than the normal control group in 6 of the 8 testing conditions. For the other three patient groups ("MS normals", MS left OND, MS right OND groups) the mean N70-P100 values 
TABLE 3

Summary of ANOVA results showing $F$ test values and significance levels for all main effects

\begin{tabular}{|c|c|c|c|c|c|}
\hline \multicolumn{6}{|c|}{ CHECK Stimulation } \\
\hline \multicolumn{3}{|c|}{ LEFT EYE } & \multicolumn{3}{|c|}{ RIGHT EYE } \\
\hline $\begin{array}{l}\text { Electrode } \\
\text { site }\end{array}$ & $\begin{array}{c}\text { VEP } \\
\text { Component }\end{array}$ & F & $\begin{array}{l}\text { Electrode } \\
\text { site }\end{array}$ & $\begin{array}{c}\text { VEP } \\
\text { Component }\end{array}$ & $\mathbf{F}$ \\
\hline $0_{1}$ & $\begin{array}{c}\text { N70 } \\
\text { P100 } \\
\text { N70-P100 }\end{array}$ & $\begin{array}{r}14.6 \$ \\
17.8 \$ \\
2.5^{*}\end{array}$ & $0_{1}$ & $\begin{array}{c}\text { N70 } \\
\text { P100 } \\
\text { N70-P100 }\end{array}$ & $\begin{array}{c}13.3 \$ \\
17.8 \$ \\
2.7^{*}\end{array}$ \\
\hline $0_{2}$ & $\begin{array}{c}\text { N70 } \\
\text { P100 } \\
\text { N70-P } 100\end{array}$ & $\begin{array}{r}17.0 \$ \\
21.1 \$ \\
2.7^{*}\end{array}$ & $\mathrm{O}_{2}$ & $\begin{array}{c}\text { N70 } \\
\text { P100 } \\
\text { N70-P100 }\end{array}$ & $\begin{array}{r}12.8 \$ \\
18.1 \$ \\
2.9+\end{array}$ \\
\hline \multicolumn{6}{|c|}{ DIAMOND Stimulation } \\
\hline & LEFT EYE & & & RIGHT EYE & \\
\hline $\begin{array}{l}\text { Electrode } \\
\text { site }\end{array}$ & $\begin{array}{c}\text { VEP } \\
\text { Component }\end{array}$ & $\mathbf{F}$ & $\begin{array}{l}\text { Electrode } \\
\text { site }\end{array}$ & $\begin{array}{c}\text { VEP } \\
\text { Component }\end{array}$ & $\mathbf{F}$ \\
\hline $0_{1}$ & $\begin{array}{c}\text { N70 } \\
\text { P100 } \\
\text { N70-P } 100\end{array}$ & $\begin{array}{r}10.5 \$ \\
14.8 \$ \\
3.1+\end{array}$ & $0_{1}$ & $\begin{array}{c}\text { N70 } \\
\text { P100 } \\
\text { N70-P100 }\end{array}$ & $\begin{array}{r}15.6 \$ \\
21.9 \$ \\
2.5^{*}\end{array}$ \\
\hline $\mathrm{O}_{2}$ & $\begin{array}{c}\text { N70 } \\
\text { P100 } \\
\text { N70-P100 }\end{array}$ & $\begin{array}{l}11.9 \$ \\
18.1 \$ \\
3.7+\end{array}$ & $\mathrm{O}_{2}$ & $\begin{array}{c}\text { N70 } \\
\text { P100 } \\
\text { N70-P100 }\end{array}$ & $\begin{array}{l}14.9 \$ \\
21.5 \$ \\
1.8 \&\end{array}$ \\
\hline & .10 & .05 & $+=p<.01$ & $\mathbf{s}=\mathrm{p}<$ & \\
\hline
\end{tabular}

were not significantly greater than the normal control group mean.

\section{VEP delay and Pattern Orientation}

Within the VEP record for each recording site the N70 and $\mathrm{P} 100 \mathrm{com}$ ponents were categorized as "normal" or "delayed" if they exceeded the statistically determined $99 \%$ criterion of the normal control group values. This was done for both diamond and check pattern orientations. Bivariate frequencies were tabulated for both check and diamond pattern conditions (Tables 4 and 5).

Table 4 shows that for check pattern-reversal stimulation, between $54 \%-64 \%$ (mean $=60 \%)$ of the overall MS population have delayed VEP components. For diamond pattern-reversal stimulation between $56 \%-68 \%$ (mean $=63 \%$ ) of the MS group have delayed VEP components.

We found that for our 31 patients with clinically definite MS but no clinical signs of optic nerve disease, between $30 \%-38 \%($ mean $=34 \%)$ were delayed to checks and between $30 \%$ $44 \%$ (mean $=36 \%$ ) were delayed with diamond pattern stimulation for both the $\mathrm{N} 70$ and $\mathrm{P} 100$ components.

Table 5 shows the percentage of the MS population showing delay in the N70 and P100 components related to pattern orientation. Between $45 \%$ $57 \%($ mean $=51 \%)$ of our MS patients had significantly delayed VEPs for both pattern orientations, while between $26 \%-32 \%$ (mean $=28 \%$ ) of the MS patients had VEPs which fell within normal limits on both check and diamond conditions.

However, between 17\% - 27\% (mean $=20 \%$ ) of our MS population showed the occurrence of orientationspecific VEP delay. To establish whether the check or diamond orientation differ in their detection of VEP delay, a McNemar test for significance of changes (Siegel, 1956) was performed with a Yates correction for continuity. The McNemar chi-square values (Table 5) were non-significant for both N70 and P100 components at every recording site. This indicates that the two pattern conditions do not significantly differ in the detection of VEP delay, i.e., neither condition is superior in detecting VEP delay.

Table 4 shows the difference in the frequency of detection of VEP delay either using a check condition alone or with the addition of a diamond condition. There is an increase of about $11 \%$ (range $=6 \%-14 \%$ ) in the frequency of detection of those affected MS patients when two pattern orientations are used.

\section{DISCUSSION}

Checkerboard pattern reversal visual evoked potentials have proved useful in the confirmation of optic nerve disease in patients with multiple sclerosis in numerous laboratories. Repetitive flash stimulation gives a high but variable yield of VEP delays in MS populations and although there have been few comparative studies of flash versus patternreversal VEPs most laboratories have turned to the use of checkerboard pattern-reversal stimulation since this appears to give a more consistent readily identifiable major positive peak. Both methods of stimulation may yield complementary information about visual system disease in MS since MS probably affects both luminance and pattern specific input channels in the visual system. Halliday et al, (1972) claimed that over $93 \%$ of patients with MS had delayed pattern VEP even when there was no clinical history of visual disturbance, but other investigators using similar sample sizes and abnormality criteria have failed to confirm such a high percentage of positive findings. The discrepant results are possibly due to differences in the categorization of the MS patient populations into those with or without a history of previous optic neuritis or as "definite" or "suspect" cases in terms of criteria used to establish the diagnosis, or due to differences in stimulating and recording conditions. There is no doubt that the stimulus luminance affects the latency of the response obtained (Cant et al, 1978) and the spatial frequency of the pattern presented causes changes in both amplitude and latency of the VEP response (Harter and White, 1970). 
TABLE 4

Percentage of delayed VEPs for check pattern-reversal alone and in combination with diamond stimulation

\% delayed
if using
CHECKS

\% delayed
if using
DIAMONDS

\author{
$\%$ increase \\ in delay \\ detection \\ over CHECKS \\ alone
}

\section{LEFT EYE}

\section{$0, N 70$}

$0_{1} \mathrm{P} 100$

$\mathrm{O}_{2} \mathrm{~N} 70$

$\mathrm{O}_{2} \mathrm{P} 100$

\section{RIGHT EYE}

$0, N 70$

$0_{1} \mathrm{P} 100$

$\mathrm{O}_{2} \mathrm{~N} 70$

$\mathrm{O}_{2} \mathrm{P} 100$

Mean

$56 \%$
$64 \%$
$58 \%$
$64 \%$

$55 \%$
$64 \%$
$58 \%$
$59 \%$
$60 \%$

$56 \%$

$58 \%$

$64 \%$

$55 \%$

$64 \%$

$58 \%$

$60 \%$

$61 \%$
$68 \%$
$56 \%$
$66 \%$
$62 \%$
$66 \%$
$61 \%$
$65 \%$
$63 \%$

$61 \%$

$68 \%$
$56 \%$

$66 \%$

$62 \%$

$66 \%$

$61 \%$

$65 \%$

$63 \%$

if using
CHECKS and
DIAMONDS

$6 \%$

$11 \%$

$9 \%$

$10 \%$

$16 \%$

$8 \%$

$14 \%$

$12 \%$

$11 \%$

TABLE 5

Percent of occurrence of VEP delay as a function of pattern orientation in the $M S$ population $(N=105)$

\begin{tabular}{lcccc}
\hline & $\begin{array}{c}\text { VEP normal } \\
\text { in both } \\
\text { orientations }\end{array}$ & $\begin{array}{c}\text { VEP delayed } \\
\text { only in one } \\
\text { orientation }\end{array}$ & $\begin{array}{c}\text { VEP delayed } \\
\text { in both } \\
\text { orientations }\end{array}$ & $\begin{array}{c}\text { McNemar } \\
\text { chi }^{2}\end{array}$ \\
LEFT EYE & $28 \%$ & $24 \%$ & $48 \%$ & 0.3 \\
$0_{1}$ N70 & $26 \%$ & $17 \%$ & $57 \%$ & 0.5 \\
$0_{1}$ P 100 & $33 \%$ & $19 \%$ & $48 \%$ & 0.1 \\
$0_{2}$ N70 & $26 \%$ & $18 \%$ & $56 \%$ & 0.2 \\
$0_{2}$ P 100 & $28 \%$ & $27 \%$ & $45 \%$ & 1.8 \\
RIG HT EYE & $28 \%$ & $27 \%$ & $45 \%$ & 0.1 \\
$0_{1}$ N70 & $27 \%$ & $25 \%$ & $48 \%$ & 0.4 \\
O $_{1}$ P 100 & $29 \%$ & $19 \%$ & $52 \%$ & 1.3 \\
$0_{2}$ N70 & $28 \%$ & $20 \%$ & $51 \%$ & \\
$0_{2}$ P 100 & & & & \\
Mean & & & &
\end{tabular}

Search for an optimal stimulus pattern for use in clinical laboratories to detect subclinical visual system deficits in MS suspect cases led Ochs and Aminoff (1980) to use a circular grating of concentric annuli. They sought to provide a stimulus which would give an equal physiological effect across the visual field and thus produce a VEP larger than that produced by check stimulation. However, they found that the VEP recorded in this way was very variable in amplitude and that intersubject variation rendered the test useless for clinical purposes. Other investigators have used bar gratings as a stimulus and this method compares favourably with the use of checkerboard phasereversing patterns (Parker and Salzen, 1977).

There is controversy whether cells in the visual cortex should be considered as bar and edge feature detectors or as spatial frequency filters which respond to the two dimensional Fourier components of patterns. Single unit studies gave evidence of hierarchically organised feature detecting mechanisms in the visual system of cat and primate suggesting that neural units were tuned to specific features in the environment (Hubel and Weisel, 1965) but DeValois et al (1979) found strong evidence for the spatial frequency model when recording single-unit responses in macaque and cat visual cortex to stimulation by gratings and check patterns of different orientations. They concluded that cortical cells respond to the orientation of the fundamental Fourier components of the pattern and not to that of the edges. Whether the human visual system responds to edges or to Fourier component orientation is secondary to the fact that orientation specificity is important to both models.

Our study confirms the presence of significant peak latency delay in the early N70 and later P100 components of the VEP to check pattern-reversal stimulation in about $60 \%$ of the population of patients with clinically definite multiple sclerosis. In addition, we report here significant VEP component delay in $63 \%$ of these patients using a diamond pattern produced by oblique orientation of the same check pattern stimulus. Our finding of only approximately $60 \%-63 \%$ of the MS population having VEP delay to pattern stimulation of one orientation (check or diamond) is probably a better estimate of the real incidence of VEP delay in MS as compared to the $97 \%$ incidence of VEP delay reported by Halliday (1977) and our figure agrees more closely with other reports in the literature. Certainly VEP delay is not the infallible test for visual system disease that is often suggested by the literature. We agree with Shahrokhi et al (1978) who reported in their MS 
population that even after definite optic neuritis the VEP latency could recover to within normal limits.

The two unilateral OND group mean latencies were significantly prolonged on the affected side and did not differ from normal on the unaffected side. The bilateral OND group mean VEP latencies were significantly prolonged for check and diamond stimulation to either eye.

The 31 patients with no clinical evidence of optic nerve disease, the "MS Normals", had a significantly delayed mean P100 latency indicating the presence of clinically silent lesions of the optic nerves or visual pathways. About $35 \%$ of our sample of "MS Normals" were abnormally delayed to check or diamond pattern stimulation. This value is far below Halliday's (1977) claim of VEP delay in $57 \%$ of those patients with no history of visual impairment and with normal disc appearance. These disparities could be accounted for by our substantially larger sample size and our more rigorous criteria for defining normal clinical visual function. Consequently we believe that our finding of VEP delay in $35 \%$ of "MS Normals" more accurately reflects the true incidence of clinically silent lesions in MS.

Our failure to find significant increases in VEP temporal dispersion in all groups other than the bilateral OND group probably reflects the fact that while single demyelinating lesions do not necessarily affect temporal dispersion per se, repeated attacks over the course of the disease eventually produce significant increase in temporal dispersion of the VEP components resulting in changes in waveform appearance. Generally speaking, members of the bilateral OND group had visual function more severely affected than members of the other $3 \mathrm{MS}$ groups and their corresponding VEPs were more markedly abnormal in appearance. We found increased temporal dispersion in 9 of 12 Friedreich's ataxia patients studied (Kirkham and Coupland, 1981b) but in those patients the appearance of this abnormality was not related to the clinical severity of the disease.
Although changing a check pattern to a diamond pattern orientation did not significantly increase the overall frequency of VEP delay in our 105 MS patients (Table 4), the addition of the diamond stimulation condition did increase the yield of MS patients with VEP delay by about $11 \%$ (Table 4 ) due to the presence of orientation-specific abnormalities in the VEPs of some MS patients. Our MS patient groups do not show a significant tendency to have orientation-specific VEP delay (i.e. normal VEP in one orientation and delayed VEP in the other orientation) as evidenced by the failure of the McNemar chi-square to reach significance (see Table 5). However, the incidence of orientation-specific VEP deficits is about $20 \%$ (range $=17 \%$ $27 \%$ ) in our 105 MS patients which clearly suggests that demyelination of the visual pathways can indeed preferentially affect some channels serving specific meridians while leaving other channels (serving other orientations) unaffected. Regan et al (1980) have established orientation-specific loss in contrast sensitivity using sinusoidal bar gratings in MS patients. Likewise, Camisa et al (1981) have reported orientation-specific VEP delay in MS patients using vertical, horizontal, and obliquely oriented bar gratings. However, our findings of orientationspecific VEP delay in MS are interesting because we have found these deficits using the standard checkerboard pattern which is routinely used in most clinical testing laboratories. Since for the diamond condition the pattern orientation is simply changed through a 45 degree rotation of the TV stimulator, this procedure can be immediately implemented in any laboratory equipped to record check pattern VEPs. It has been our experience that the increased diagnostic yield (by some $11 \%$ ) renders the additional few minutes testing time worthwhile.

\section{ACKNOWLEDGEMENTS}

Dr. S.G. Coupland is a Post Doctoral Fellow supported by the Multiple Sclerosis Society of Canada. This work was aided by a grant from the Canadian National Institute for the Blind out of the E.A. Baker Foundation. The Stephen
B. Roman Foundation and the W. Garfield Weston Foundation and The Town of Mount Royal Old Timers Executive Hockey League gave generous assistance towards the establishment of our visual neurophysiological laboratory. Ms. Judy Bengle R.N. gave competent technical assistance.

\section{REFERENCES}

BODIS-WOLLNER, I., HENDLEY, C.D., MYLIN, L.H., THORNTON, J. (1979). Visual evoked potentials and the visuogram in multiple sclerosis. Ann. Neurol. 5: 40-47.

CAMISA, J., MYLIN, L.H. and BODISWOLLNER, I. (1981). The effect of stimulus orientation on the visual evoked potential in multiple sclerosis. Ann. Neurol. 10: $532-539$.

CANT, B.R., HUME, A.L. and SHAW, N.A. (1978). Effects of luminance on the pattern visual evoked potential in multiple sclerosis. Electroenceph. Clin. Neurophysiol. 45: 496504.

COUPLAND, S.G. and KIRKHAM, T.H. (1982). Flash electroretinogram abnormalities in patients with clinically definite multiple sclerosis. Canad. J. Neurol. Sci. 9: 325-330.

DEVALOIS, K.K., DEVALOIS, R.L. and YUND, E.W. (1979). Response of striate cortical cells to grating and checkerboard patterns. J. Physiol. 291: 483-505.

HALLIDAY, A.M., McDONALD, W.I. and MUSHIN, J. (1972). Delayed visual evoked responses in optic neuritis. Lancet 1: $982-$ 985.

HALLIDAY, A.M. (1981). Visual evoked potentials in demyelinating disease. In Demyelinating disease: basic and clinical electrophysiology. Ed. Waxman, S.G. and Ritchie, J.M. Raven Press. New York, pp. 201-215.

HARTER, M.R. and WHITE, C.T. (1968). Effects of contour sharpness and check size on visually evoked potentials. Vision Res. 8: 701-711.

HUBEL, D.H. and WIESEL, T.N. (1968). Receptive fields and functional architecture of monkey striate cortex. J. Physiol. 195: 215-225.

KIRKHAM, T.H. and COUPLAND, S.G. (1981a). Multiple regression analysis of diagnostic predictors in optic nerve disease. Canad. J. Neurol. Sci. 8: 67-72.

KIRKHAM, T.H. and COUPLAND, S.G. (198lb). An electroretinal and visual evoked potential study in Friedreich's ataxia. Canad. J. Neurol. Sci. 8: 289-294. 
McDONALD, W.I. and HALLIDAY, A.M. (1977). Diagnosis and classification of multiple sclerosis. Brit. Med. Bull. 33: 4-8.

OCHS, A.L. and AMINOFF, M.J. (1980). Visual evoked potentials elicited by circular grating. Arch. Neurol. 37: 308-309.
PARKER, D.M. and SALZEN, E.A. (1977). Latency changes in the human visual evoked response to sinusoidal gratings. Vision Res. 18: 1201-1204.

REGAN, D., WHITLOCK, J.A., MURRAY, T.J. and BEVERLEY, K.I. (1980). Orientation specific losses of contrast sensitivity in multiple sclerosis. Invest. Ophthalmol. Vis. Sci. 19: 324-328.
SHAHROK HI, F., CHIAPPA, K.H. and YOUNG, R.W. (1978). Pattern shift visual evoked responses. Two hundred patients with optic neuritis and/or multiple sclerosis. Arch. Neurol. 35: 65-71.

SIEGEL, S. (1956). Nonparametric statistics for the behavioral sciences. McGraw-Hill Publishers, New York. 